\title{
Paediatric Autoimmune Neuropsychiatric Disorder Associated with Group A Beta-Haemolytic Streptococcal Infection: An Indication for Tonsillectomy? A Review of the Literature
}

\author{
Amarkumar Dhirajlal Rajgor $\mathbb{D}^{1},{ }^{1}$ Navid Akhtar Hakim, ${ }^{1}$ Sanah Ali, ${ }^{2}$ and Adnan Darr ${ }^{1}$ \\ ${ }^{1}$ University Hospital North Midlands, Royal Stoke University Hospital, Stoke-on-Trent, UK \\ ${ }^{2}$ Russells Hall Hospital, Dudley, UK \\ Correspondence should be addressed to Amarkumar Dhirajlal Rajgor; drarajgor@gmail.com
}

Received 13 August 2017; Revised 20 November 2017; Accepted 3 December 2017; Published 21 February 2018

Academic Editor: Sergio Motta

Copyright (C) 2018 Amarkumar Dhirajlal Rajgor et al. This is an open access article distributed under the Creative Commons Attribution License, which permits unrestricted use, distribution, and reproduction in any medium, provided the original work is properly cited.

\begin{abstract}
Background. Paediatric Autoimmune Neuropsychiatric Disorder Associated with Streptococcal Infection (PANDAS) is the acute onset of neuropsychiatric symptoms following group A beta-haemolytic streptococcal infection. The aetiology remains elusive. However, with group A streptococcus being the most common bacterial cause of tonsillitis, surgical intervention in the form of tonsillectomy has often been considered as a potential therapy. Methods. A MEDLINE ${ }^{\circledR}$ search was undertaken using keywords "PANDAS" or "paediatric autoimmune neuropsychiatric disorders associated with streptococcus" combined with "tonsillectomy". Results. Six case reports and 3 case series met the inclusion criteria. Demesh et al. (case series) reported a dramatic reduction in neuropsychiatric symptom severity in the patient cohort undergoing tonsillectomy. Two case series suggest that there is no association between tonsillectomy and resolution of PANDAS. Conclusion. Due to the lack of uniform data and sporadic reports, tonsillectomy should be carefully adopted for the treatment of this disorder. In particular, tonsillectomies/adenoidectomies to alleviate neuropsychiatric symptoms should be avoided until more definitive evidence is at our disposal. This review highlights the importance of a potential collaborative prospective study.
\end{abstract}

\section{Introduction}

Group A beta-haemolytic streptococcal (GABHS) tonsillitis, more frequently known as streptococcal pharyngitis, is highly prevalent in children especially in those who are between the ages of 5 and 15 years [1]. It is the commonest bacterial cause of tonsillitis in both children and adults, accounting for approximately $30 \%$ of all cases [2], with at least a single infection before the age of 5 [3]. Furthermore, several studies have suggested that as many as half of children presenting with pharyngitis could be GABHS carriers, making virtuously impossible to distinguish these children from those with the actual disease just based on signs and symptoms alone [4-9].

Paediatric Autoimmune Neuropsychiatric Disorder Associated with Streptococcal Infection (PANDAS) is the acute onset of neuropsychiatric symptoms following GABHS infection. PANDAS was initially described by Swedo et al. in 1998 following a study of 50 patients who had developed obsessive-compulsive disorder (OCD) and/or tics following a poststreptococcal autoimmune process $[10,11]$. Despite much research, the aetiology of PANDAS is still unclear, with several hypotheses suggesting a variety of genetic and environmental factors as well as autoimmune mechanisms [12]. The incidence and prevalence of PANDAS remain uncertain [13] but some studies suggest that it could play a role in more than $10 \%$ of childhood-onset OCD [14] and tics [15].

Group A streptococcal infection pathogenesis is described in three stages $[16,17]$. Initially there is adherence to the pharyngeal epithelium where pili-like cell surface structures play a key role in pharyngeal epithelial cell adherence 
and biofilm formation [18, 19]. Next, nutrients based on carbon sources are attained for proliferation, which triggers the clinical features found in pharyngitis $[20,21]$. This is followed by group A streptococcus being able to avoid the host's innate immune response by secreting proteins that inhibits phagocytosis and polymorphonuclear- (PMN-) mediated killing $[22,23]$. Evidence suggests that PANDAS is most probably an autoimmune phenomenon involving pathogenic autoantibodies [24]. However, to date, the biological value of detected antineuronal antibodies remains to be determined $[10,25]$. The commonest hypothesis is that of molecular mimicry where it is thought the autoantibodies that target brain structures are abnormally produced $[1,2]$. Other mechanisms such as epitope spreading and bystander activation also seem to play a role in the production of defective autoantibody production $[4,5,26]$. Human basal ganglia antigens have been demonstrated in patients with encephalitis and movement disorders in 2004 [27] but the clinical relevance was not widely accepted due to methods used (western blotting of soluble brain extracts) [28]. Subsequently an intracellular enzyme was identified as a target antigen of these antibasal ganglia autoantibodies in 2006 [29]. In 2012, it was reported that both dopamine-1 receptor (D1R) and dopamine-2 receptor (D2R) antibodies were detected in patients with Sydenham's chorea and PANDAS [30] but methods used (ELISA and western blotting) were again not optimal for cell surface antigen detection [31]. These antibodies bind to the neuronal surface and cause elevated calcium/calmodulin-dependent protein kinase II signalling in neuronal cell lines [26]. Dale et al. later implemented a flow-cytometry based approach to identify autoantibodies against neuronal surface antigenic targets as this was a more specific methodology, but none were detected in the PANDAS group [32].

Tonsillitis remains a clinical diagnosis. To distinguish between a viral and a bacterial origin, laboratory testing such as throat swab culture, rapid antigen detection testing (RADT), or antistreptolysin titres (ASOT) is necessary. If performed correctly, throat swab cultures grown on a blood agar plate have a sensitivity of $90-95 \%$ in detecting GABHS [33]. This remains the standard test used despite the disadvantage of time constraints for results analysis [34]. RADT is faster but more expensive, with a specificity of $95 \%$, but a sensitivity of $80-90 \%$ [35]. This means there is a chance of false negative results with RADT, which could result in misdiagnosis and group A streptococcal spread and even rarely lead to an increased risk of suppurative and nonsuppurative complications [36]. Antibodies against streptokinase (ASK), streptolysin O (ASO), and deoxyribonuclease $\mathrm{B}$ (anti-DNAseB) are not reliable markers for acute GABHS infection, used either alone or in combination with one another [37]. According to the National Institute of Mental Health Clinical Diagnostic Criteria for PANDAS, the diagnosis of PANDAS is based on five criteria (presence of OCD and/or a tic disorder, prepubertal onset, abrupt onset and relapsing-remitting course, association with GABHS infection, and association with neurological abnormalities) $[10,38-41]$.

\section{The Role of Surgical Intervention in PANDAS}

Surgical intervention by means of tonsillectomy or adenoidectomy has long been considered as an alternative to pharmacological therapy in the management of PANDAS [42]. Literature advocating such management appears to have increased despite the ongoing need for robust evidence. Despite the fact that many PANDAS patients meet the globally acknowledged criteria for tonsillectomy ( $\geq 7$ severe episodes of tonsillitis within the preceding year, $\geq 5$ within the preceding 2 years or, $\geq 3$ within the preceding 3 -year period) and therefore PANDAS generally represents a comorbidity in these cases, a great deal of disagreement as to the efficacy of this treatment option in providing remission or symptoms improvement still exists due to insufficient evidence [42]. The role of tonsillectomy in PANDAS has predominantly been evoked by case reports citing a reduction in frequency of tics as well as OCD after surgery $[43,44]$.

Three case series and six case reports met the inclusion criteria. The results are summarized in Tables 1 and 2, respectively.

\section{Method}

A review of the literature was performed to evaluate the published evidence regarding surgical management (tonsillectomy) of PANDAS. A structured search of the MEDLINE online database (no interval/period was stipulated) was undertaken. Keywords "PANDAS" or "paediatric autoimmune neuropsychiatric disorders associated with streptococcus" were combined with "tonsillectomy". The criteria for inclusion of citations were English language case reports and case series with confirmed and definitive cases of PANDAS according to the Institute of Mental Health Clinical Diagnostic Criteria for PANDAS. References of all the subsequent articles including case series were also evaluated for further case reports relevant to the subject. Information including clinical presentation, ASOT titre, medication attempted, follow-up time, and overall outcome was recorded from the details provided in each case report.

\section{Discussion}

With regard to case series, Murphy et al. [45] played a crucial role in refuting the notion that tonsillectomy may play a protective role in the development of the disease. Using the DSM-IV-R criteria for OCD/tic disorders, 112 patients were included in a prospective study to ascertain if a correlation existed between ASO titres in surgical patients $(n=36)$, as well as symptomology. Of the 112 patients, 43 had confirmed PANDAS. More than $50 \%$ of cases developed neuropsychiatric symptoms within 2.4-2.9 years after tonsillectomy. Also, the resultant incidence of PANDAS was almost twice that of the nonsurgical cohort. Interestingly, $46.5 \%$ of the PANDAS population underwent surgery compared to only $23.2 \%$ of the non-PANDAS population. Although PANDAS is not an indication for tonsillectomy, this may demonstrate the 


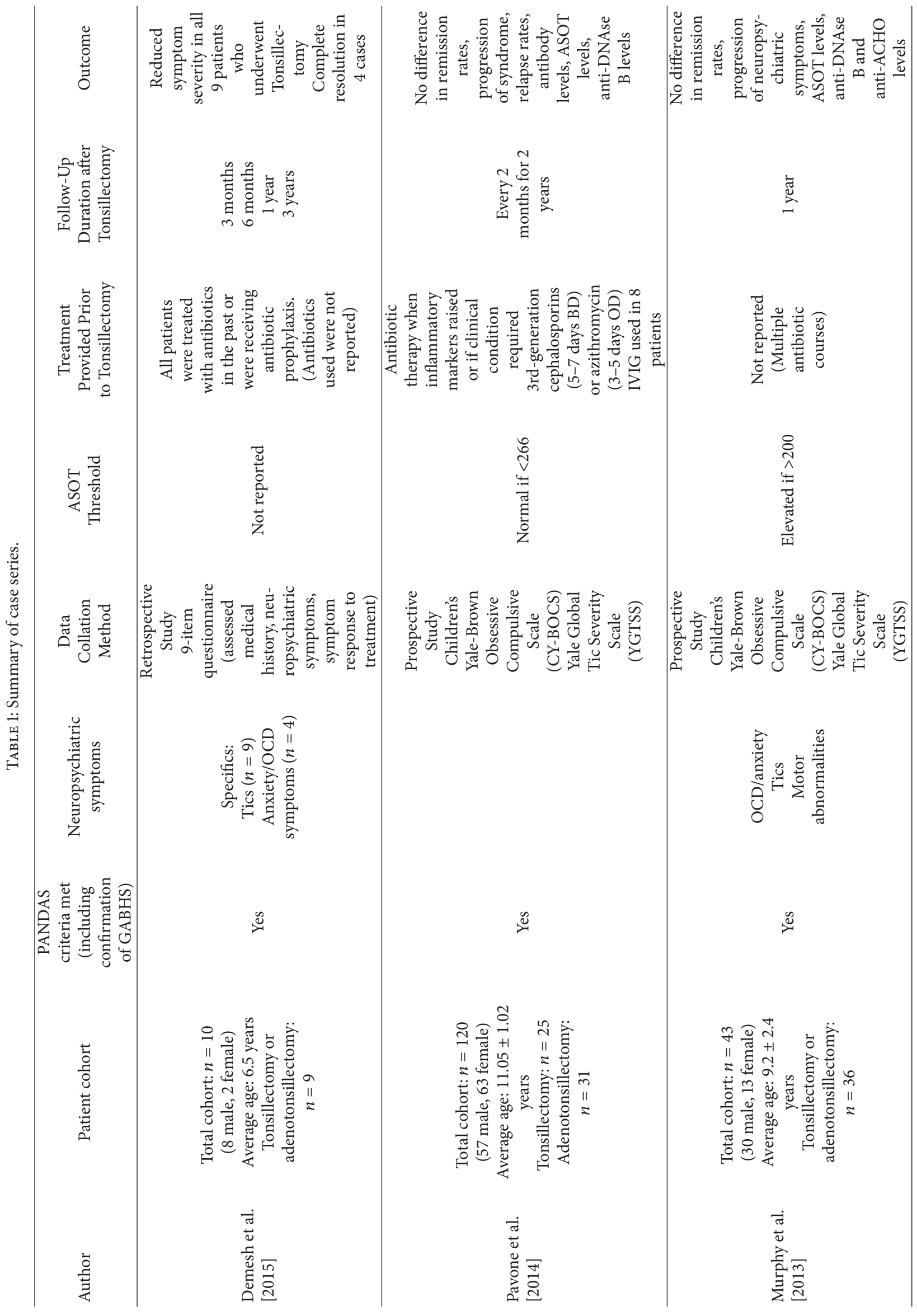




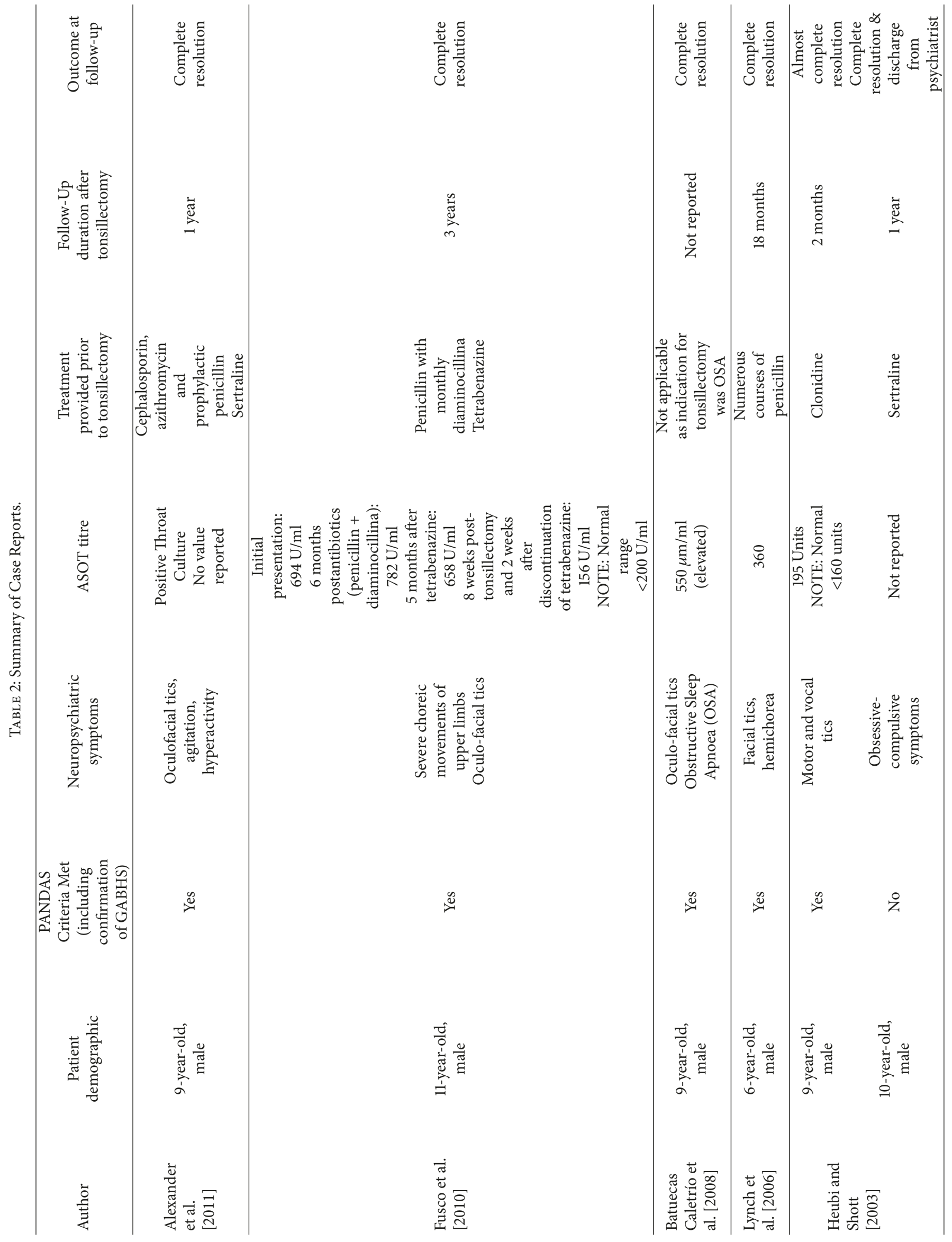




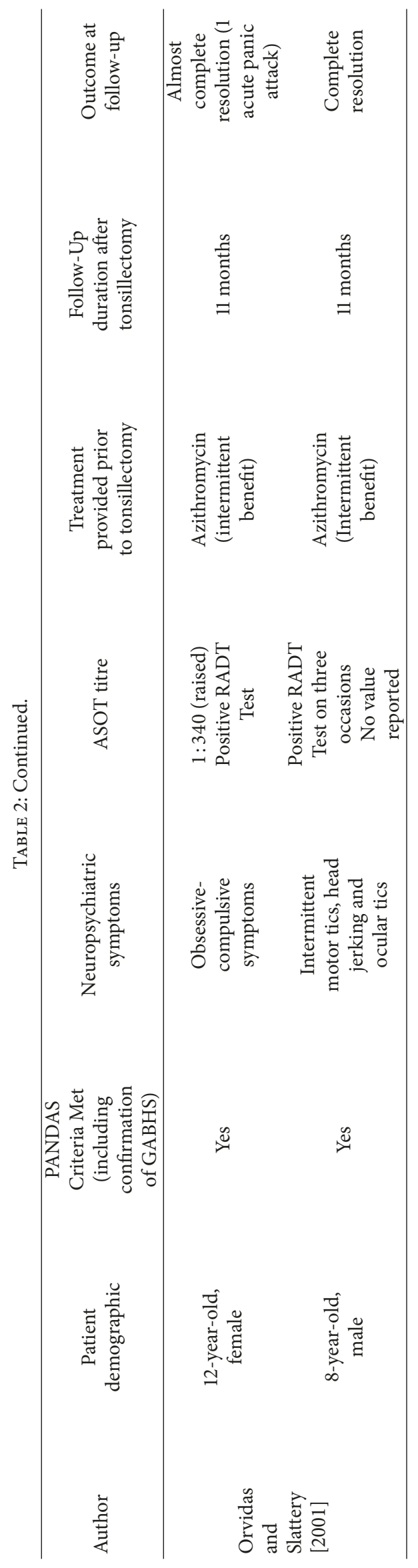


potential influence that neuropsychiatric symptoms may have on a clinicians' decision to operate. This study has numerous limitations. The study design only included children with current neuropsychiatric symptoms that were retrospectively stratified based on whether a tonsillectomy was performed. Therefore, in case of postoperative remission, a child would not have entered the study. In addition, the ASO titres were assessed at the beginning of the study rather than verifying a temporal relationship with $\mathrm{OCD} /$ tic occurrence. ASO titres have been shown to vary with age and are more representative of an infection if serial measures are performed [46]; thus it would have been more valuable to undertake serial titres and adjust them for age.

Another prospective nonrandomized case series was reported by Pavone et al. (2014) [47]. The study included 120 patients with PANDAS who either had undergone tonsillectomy or adenotonsillectomy $(n=56)$ or had been managed conservatively $(n=64)$. Patients were followed up for 24 months. Clinical progression, antibody production, and neuropsychiatric symptomatology did not differ in the two groups. Importantly, all assessments of symptoms were made using the validated Children's Yale-Brown Obsessive Compulsive Scale (CY-BOCS) and Yale Global Tic Severity Scale (YDTSS) by appropriately trained clinicians. However, Pavone et al. did not account for the several factors that can influence titres such as age. Both the antibody levels and ASO titres can be elevated for months and thus the utilisation of a single time point as in this study provides very limited information regarding the streptococcal infection.

Perhaps the most comprehensive case series presented to date was a retrospective study conducted by Demesh et al. (2015) where 10 patients met strict diagnostic criteria for PANDAS [42]. Comparisons were made between parental reports of symptom severity at diagnosis, after antibiotic treatment $(n=10)$ and after tonsillectomy $(n=9)$ using a baseline severity score. Symptom severity improved at all periods after tonsillectomy compared with antibiotics alone. In the surgical cohort, 4 out of nine cases demonstrated a complete resolution, further adding weight to the belief that patients suboptimally treated with antibiotic therapy may benefit from tonsillectomy. However, this study would be subject to recall bias as information was extracted retrospectively from the parents' historical accounts of symptoms using a nonvalidated symptom severity score. Additionally, as the parents had consented on behalf of their child, they would potentially have positively skewed perceptions of the outcome.

In terms of individual cases, Alexander et al. [48] described the case of a 9-year-old boy presenting with ocular facial tics, agitation, and hyperactivity following multiple recurrent GABHS infections. At a routine one-year followup following a bilateral tonsillectomy, no further recurrent GABHS infections were reported, and most notably there was a complete resolution of his previous neuropsychiatric symptoms [48]. However, this child was also treated with sertraline prior to tonsillectomy but the report does not clarify whether this medical therapy was stopped after surgery. Hence, outcome may have been the natural progression of the disease in a patient who continued with sertraline for a longer duration of time. Similarly, Batuecas Caletrío et al. [49] described a child with PANDAS (facial tics), following recurrent GABHS, who again, following tonsillectomy, demonstrated a complete resolution of his neuropsychiatric symptoms. Batuecas Caletrío et al. stated that the child had "regular checks" but does not mention the duration of follow-up, thus making it difficult to conclude whether remission occurred instantly or two years later.

Fusco et al. presented the case of an 11-year-old boy, who developed severe choreic movements and satisfied the criteria for the diagnosis of PANDAS [50]. Although the paper reported a complete remission in neurological symptomology following tonsillectomy, cessation in neurological symptomology could not be purely linked to surgical intervention due to the initial initiation of twice-daily tetrabenazine and the long-term use of prophylactic antibiotics. ASO titres levels were reported to be in the normal range following surgical intervention, but, as previously described, the reliability of this finding remains doubtful [37].

Orvidas and Slattery (2001) [51] presented the first case of PANDAS in siblings, with recurrent GABHS pharyngitis despite antibiotic treatment. The presenting complaint was behavioral alterations, mainly tics and OCS, coexistent with recurrent infective bouts. Following tonsillectomy, both siblings exhibited a marked improvement in GAS pharyngitis recurrences and OCS/tic exacerbation at 11-month followup. Similarly, Heubi and Shott (2003) reviewed the case of two brothers, one with OCD and the other with a tic disorder, both of whom improved significantly after undergoing adenotonsillectomy for treatment of their recurrent tonsillitis [52]. Doubts regarding the underlying diagnosis, principally due to minimally raised ASO levels in one of the two cases, and an absence of the behavioural oscillations crucially linked to PANDAS therefore question the reliability of the diagnosis.

\section{Limitations of Literature}

There are numerous generic limitations to this sparse literature. First of all, there are incomplete data in several reports regarding the timespan to complete resolution. For example, Bautecus et al. states complete resolution at "regular checks" which provides no relevant information regarding the timespan to complete resolution. Secondly, for the majority of these cases, single measurements of ASO titres and antibody levels were conducted which provide limited information on streptococcal infections. Additionally, the studies utilised various laboratories measures with different upper limits and thus could make drawing conclusions more difficult. The concomitant use of other medical therapy such as antibiotics and neuropsychiatric drugs makes it difficult to state surgery as the sole reason for PANDAS resolution. Furthermore, none of the studies provided information regarding the duration and severity of the neuropsychiatric symptoms prior to intervention.

\section{Conclusion}

PANDAS has become an evolving area of research for a possible relationship, investigating the relationship between 
GABHS infections in children and the development of either an obsessive-compulsive disorder or tic disorder. Overall, the limited series and case reports in the literature provide an unsubstantiated link between tonsillectomy and symptom remission, predominantly as other notable variables including pharmacological therapy (antibiotics and tetrabenazine), and stress/fatigue were not accounted for. Although deemed as relatively small risk procedure, complications of tonsillectomy must also be considered into the debate advocating surgical intervention. Therefore adenotonsillectomy as a potential therapeutic strategy may only be appropriate in children with antibiotic-refractory PANDAS.

PANDAS is a significantly debilitating condition, with detrimental effects on a child's psychophysical development. This review provides an insight into the current literature and the potential for surgery to treat PANDAS. However due to the current limitations of the literature drawing definitive conclusions is difficult. Thus, the next required steps would involve conducting a prospective collaborative study.

\section{Conflicts of Interest}

There are no conflicts of interest regarding the publication of this paper.

\section{Authors' Contributions}

Amarkumar Dhirajlal Rajgor contributed to original idea of research, writing all sections of the paper (Abstract, Background, Methods, Results, and Conclusion), and literature search. Navid Akhtar Hakim assisted in the input in all sections of the paper (Abstract, Background, Methods, Results, and Conclusion), literature search, and review following completion. Sanah Ali helped in input in all sections of the paper (Abstract, Background, Methods, Results, and Conclusion) and review following completion. Adnan Darr, supervisor, contributed to original idea of research, input, and review of all sections of the paper.

\section{References}

[1] B. A. Choby, "Diagnosis and treatment of streptococcal pharyngitis," Fam Physician, vol. 79, pp. 383-390, 2009.

[2] A. Barzilai, D. Miron, and S. Sela, "Etiology and management of acute and recurrent group a Streptococcal tonsillitis," Current Infectious Disease Reports, vol. 3, no. 3, pp. 217-223, 2001.

[3] A. Efstratiou and T. Lamagni, Epidemiology of Streptococcus pyogenes, 2016, Available from URL: https://www.ncbi.nlm.nih .gov/books/NBK343616/.

[4] N. Shaikh, E. Leonard, and J. M. Martin, "Prevalence of streptococcal pharyngitis and streptococcal carriage in children: A meta-analysis," Pediatrics, vol. 126, no. 3, pp. e557-e564, 2010.

[5] M. H. Danchin, S. Rogers, and G. Selvaraj, “The burden of group A streptococcal pharyngitis in Melbourne families," Indian J Med Res, pp. 119-144, 2004.

[6] A. Kunnamo, M. Korppi, and M. Helminen, "Tonsillitis in children: unnecessary laboratory studies and antibiotic use," World Journal of Pediatrics, vol. 12, no. 1, pp. 114-117, 2016.
[7] S. Lin, E. L. Kaplan, X. Rao et al., "A school-based program for control of group A streptococcal upper respiratory tract infections: A controlled trial in Southern China," The Pediatric Infectious Disease Journal, vol. 27, no. 8, pp. 753-755, 2008.

[8] J. M. Martin, M. Green, K. A. Barbadora, and E. R. Wald, “Group a streptococci among school-aged children: Clinical characteristics and the carrier state," Pediatrics, vol. 114, no. 5, pp. 12121219, 2004.

[9] N. Principi, P. Marchisio, and A. Calanchi, "Drugs ExpClin Res," Drugs ExpClin Res, vol. 16, no. 12, pp. 639-647, 1990.

[10] S. E. Swedo, H. L. Leonard, and M. Garvey, "Paediatric autoimmune neuropsychiatric disorders associated with streptococcal infections: clinical description of the first 50 cases," Am J Psychiatry, vol. 155, pp. 264-271, 1998.

[11] R. Kurlan and E. L. Kaplan, "The pediatric autoimmune disorders associated with streptococcal infection (PANDAS) etiology for tics and obsessive-compulsive symptoms: hypothesis or entity? practical considerations for the clinician," Pediatrics, vol. 113, no. 4, pp. 883-886, 2004.

[12] Leonard H. L and S. E. Swedo, "Paediatric autoimmune neuropsychiatric disorders associated with streptococcal infection (PANDAS)," International Journal of Neuropsychopharmacy, vol. 4, pp. 191-198, 2001.

[13] M. E. Pichichero, "The PANDAS syndrome," Hot Topics in Infection and Immunity in Children, vol. 634, pp. 205-216, 2009.

[14] J. D. Giuliano, A. Zimmerman, J. T. Walkup, and H. S. Singer, "Prevalence of pediatric autoimmune neuropsychiatric disorders associated with streptococcal infection by history in a consecutive series of community referred children evaluated for tics," Ann Neurol, vol. 44, no. 3, p. 556, 1998.

[15] F. Cardona and G. Orefici, "Group A streptococcal infections and tic disorders in an Italian pediatric population," Journal of Pediatrics, vol. 138, no. 1, pp. 71-75, 2001.

[16] R. J. Olsen, S. A. Shelburne, and J. M. Musser, "Molecular mechanisms underlying group A streptococcal pathogenesis," Cellular Microbiology, vol. 11, no. 1, pp. 1-12, 2009.

[17] A. L. Bisno, M. A. Gerber, J. M. Gwaltney, E. L. Kaplan, and R. H. Schwartz, "Practice guidelines for the diagnosis and management of group A streptococcal pharyngitis," Clinical Infectious Diseases, vol. 35, no. 2, pp. 113-125, 2002.

[18] H. S. Courtney, D. L. Hasty, and J. B. Dale, "Molecular mechanisms of adhesion, colonization, and invasion of group A streptococci," Annals of Medicine, vol. 34, no. 2, pp. 77-87, 2002.

[19] H. J. Kang, F. Coulibaly, F. Clow, T. Proft, and E. N. Baker, "Stabilizing isopeptide bonds revealed in gram-positive bacterial pilus structure," Science, vol. 318, no. 5856, pp. 1625-1628, 2007.

[20] H. Gough, G. A. Luke, J. A. Beeley, and D. A. M. Geddes, "Human salivary glucose analysis by high-performance ionexchange chromatography and pulsed amperometric detection," Archives of Oral Biolog, vol. 41, no. 2, pp. 141-145, 1996.

[21] S. A. Shelburne, D. B. Keith, M. T. Davenport, N. Horstmann, R. G. Brennan, and J. M. Musser, "Molecular characterization of group A Streptococcus maltodextrin catabolism and its role in pharyngitis," Molecular Microbiology, vol. 69, no. 2, pp. 436-452, 2008.

[22] K. Virtaneva, S. F. Porcella, M. R. Graham et al., "Longitudinal analysis of the group A Streptococcus transcriptome in experimental pharyngitis in cynomolgus macaques," Proceedings of the National Acadamy of Sciences of the United States of America, vol. 102, no. 25, pp. 9014-9019, 2005. 
[23] N. P. Hoe, R. M. Ireland, F. R. DeLeo et al., "Insight into the molecular basis of pathogen abundance: Group A Streptococcus inhibitor of complement inhibits bacterial adherence and internalization into human cells," Proceedings of the National Acadamy of Sciences of the United States of America, vol. 99, no. 11, pp. 7646-7651, 2002.

[24] M. Lim and A. Vincent, "More movements in neuroimmunology," Brain, vol. 135, no. 11, pp. 3201-3202, 2012.

[25] V. Jain, "Inflammatory and autoimmune disorders of the nervous system in children," Clinics in Developmental Medicine, pp. 184-185, 2010.

[26] C. A. Kirvan, S. E. Swedo, L. A. Snider, and M. W. Cunningham, "Antibody-mediated neuronal cell signaling in behavior and movement disorders," Journal of Neuroimmunology, vol. 179, no. 1-2, pp. 173-179, 2006.

[27] R. C. Dale, A. J. Church, R. A. H. Surtees et al., "Encephalitis lethargica syndrome: 20 new cases and evidence of basal ganglia autoimmunity," Brain, vol. 127, part 1, pp. 21-33, 2004.

[28] A. Vincent, "Encephalitis lethargica: Part of a spectrum of poststreptococcal autoimmune diseases?" Brain, vol. 127, no. 1, pp. 2-3, 2004.

[29] R. C. Dale, P. M. Candler, A. J. Church, R. Wait, J. M. Pocock, and G. Giovannoni, "Neuronal surface glycolytic enzymes are autoantigen targets in post-streptococcal autoimmune CNS disease," Journal of Neuroimmunology, vol. 172, no. 1-2, pp. 187197, 2006.

[30] L. Brimberg, I. Benhar, A. Mascaro-Blanco et al., "Behavioral, pharmacological, and immunological abnormalities after streptococcal exposure: A novel rat model of sydenham chorea and related neuropsychiatric disorders," Neuropsychopharmacology, vol. 37, no. 9, pp. 2076-2087, 2012.

[31] A. Macerollo and D. Martino, Pediatric Autoimmune Neuropsychiatric Disorders Associated with Streptococcal Infections (PANDAS): An Evolving Concept, 2013, http://tremorjournal.org/article/view/167.

[32] R. C. Dale, V. Merheb, S. Pillai et al., "Antibodies to surface dopamine-2 receptor in autoimmune movement and psychiatric disorders," Brain, vol. 135, no. 11, pp. 3453-3468, 2012.

[33] S. Felsenstein, D. Faddoul, R. Sposto, K. Batoon, C. M. Polanco, and J. D. Bard, "Molecular and clinical diagnosis of group A streptococcal pharyngitis in children," Journal of Clinical Microbiology, vol. 52, no. 11, pp. 3884-3889, 2014.

[34] W. L. Lean, S. Arnup, M. Danchin, and A. C. Steer, "Rapid diagnostic tests for group a streptococcal pharyngitis: A metaanalysis," Pediatrics, vol. 134, no. 4, pp. 771-781, 2014.

[35] J. F. Cohen, N. Bertille, R. Cohen, and M. Chalumeau, "Rapid antigen detection test for group A streptococcus in children with pharyngitis," Cochrane Database of Systematic Reviews, no. 7, Article ID CD010502, 2016.

[36] B. A. Choby, "Diagnosis and treatment of streptococcal pharyngitis, American Family Physician, vol. 79, no. 5, pp. 383-390, 2009.

[37] C. C. Blyth and P. W. Robertson, "Anti-streptococcal antibodies in the diagnosis of acute and post-streptococcal disease: streptokinase versus streptolysin O and deoxyribonuclease B," Pathology, vol. 38, no. 2, pp. 152-156, 2006.

[38] H. S. Singer, D. L. Gilbert, D. S. Wolf, J. W. Mink, and R. Kurlan, "Moving from PANDAS to CANS," Journal of Pediatrics, vol. 160, no. 5, pp. 725-731, 2012.

[39] S. E. Swedo, "From research subgroup to clinical syndrome: modifying the PANDAS criteria to describe pans (pediatric acute-onset neuropsychiatric syndrome)," Pediatrics \& Therapeutics, vol. 02, no. 02, 2012.

[40] N. Shaikh, E. Leonard, and J. M. Martin, "Prevalence of streptococcal pharyngitis and streptococcal carriage in children: A meta-analysis," Pediatrics, vol. 126, no. 3, pp. 557-564, 2010.

[41] S. E. Swedo, "Sydenham's Chorea: A Model for Childhood Autoimmune Neuropsychiatric Disorders," Journal of the American Medical Association, vol. 272, no. 22, pp. 1788-1791, 1994.

[42] D. Demesh, J. M. Virbalas, and J. P. Bent, "The role of tonsillectomy in the treatment of Pediatric Autoimmune Neuropsychiatric Disorders Associated with Streptococcal Infections (PANDAS)," JAMA Otolaryngology - Head and Neck Surgery, vol. 141, no. 3, pp. 272-275, 2015.

[43] B. W. Blakley and A. E. Magit, "The role of tonsillectomy in reducing recurrent pharyngitis: A systematic review," Otolaryngology-Head and Neck Surgery, vol. 140, no. 3, pp. 291-297, 2009.

[44] L. J. Orvidas, J. L. St. Sauver, and A. L. Weaver, "Efficacy of tonsillectomy in treatment of recurrent group A $\beta$-hemolytic streptococcal pharyngitis," The Laryngoscope, vol. 116, no. 11, pp. 1946-1950, 2006.

[45] T. K. Murphy, A. B. Lewin, E. C. Parker-Athill, E. A. Storch, and P. J. Mutch, "Tonsillectomies and adenoidectomies do not prevent the onset of pediatric autoimmune neuropsychiatric disorder associated with group a streptococcus," The Pediatric Infectious Disease Journal, vol. 32, no. 8, pp. 834-838, 2013.

[46] E. L. Kaplan, C. D. Rothermel, and D. R. Johnson, "Antistreptolysin $\mathrm{O}$ and anti-deoxyribonuclease $\mathrm{B}$ titers: normal values for children ages 2 to 12 in the United States," Pediatrics, vol.101, no. 1, part 1, pp. 86-88, 1998.

[47] P. Pavone, V. Rapisarda, A. Serra et al., "Pediatric autoimmune neuropsychiatry disorder associated with group a streptococcal infection: The role of surgical treatment," International Journal of Immunopathology and Pharmacology, vol. 27, no. 3, pp. 371378, 2014.

[48] A. A. Z. Alexander, N. J. Patel, C. A. Southammakosane, and M. M. Mortensen, "Pediatric autoimmune neuropsychiatric disorders associated with streptococcal infections (PANDAS): An indication for tonsillectomy," International Journal of Pediatric Otorhinolaryngology, vol. 75, no. 6, pp. 872-873, 2011.

[49] Á. Batuecas Caletrío, F. Sánchez González, S. S. Cruz Ruiz, P. Santos Gorjón, and P. Blanco Pérez, "PANDAS Syndrome: ANew Tonsillectomy Indication?" Acta Otorrinolaringologica (English Edition), vol. 59, no. 7, pp. 362-363, 2008.

[50] F. R. Fusco, A. Pompa, G. Bernardi et al., "A case of PANDAS treated with tetrabenazine and tonsillectomy," Journal of Child Neurology, vol. 25, no. 5, pp. 614-615, 2010.

[51] L. J. Orvidas and M. J. Slattery, "Pediatric autoimmune neuropsychiatric disorders and streptococcal infections: Role of otolaryngologist," The Laryngoscope, vol. 111, no. 9, pp. 1515-1519, 2001.

[52] C. Heubi and S. R. Shott, "PANDAS: Pediatric autoimmune neuropsychiatric disorders associated with streptococcal infections - An uncommon, but important indication for tonsillectomy," International Journal of Pediatric Otorhinolaryngology, vol. 67, no. 8, pp. 837-840, 2003. 


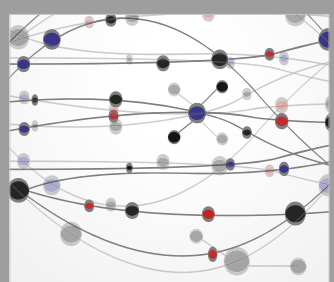

The Scientific World Journal
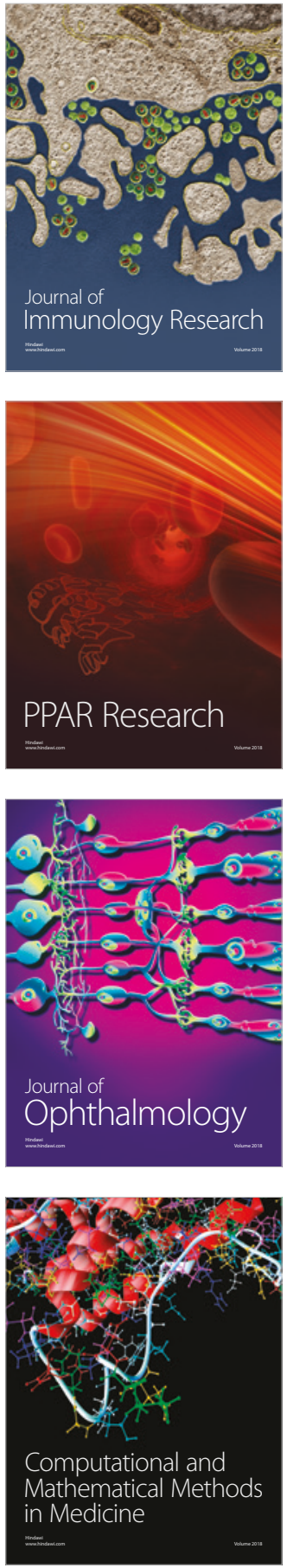

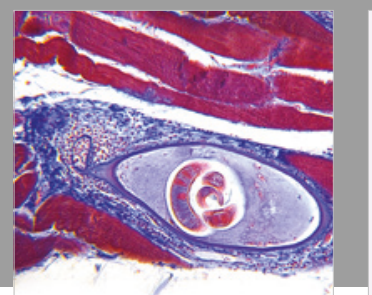

Gastroenterology Research and Practice

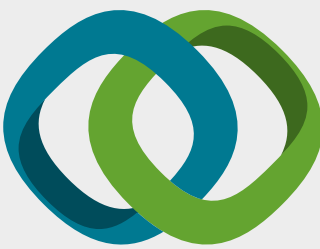

\section{Hindawi}

Submit your manuscripts at

www.hindawi.com
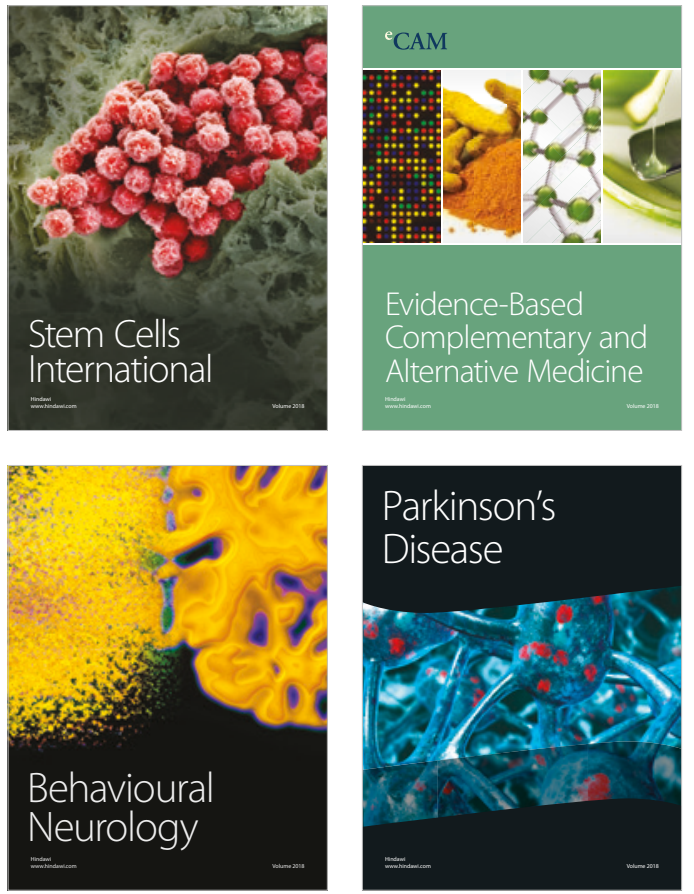

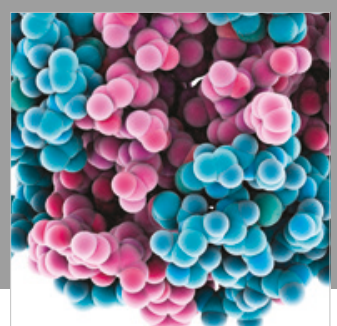

ournal of

Diabetes Research

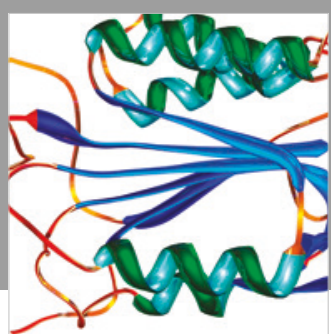

Disease Markers
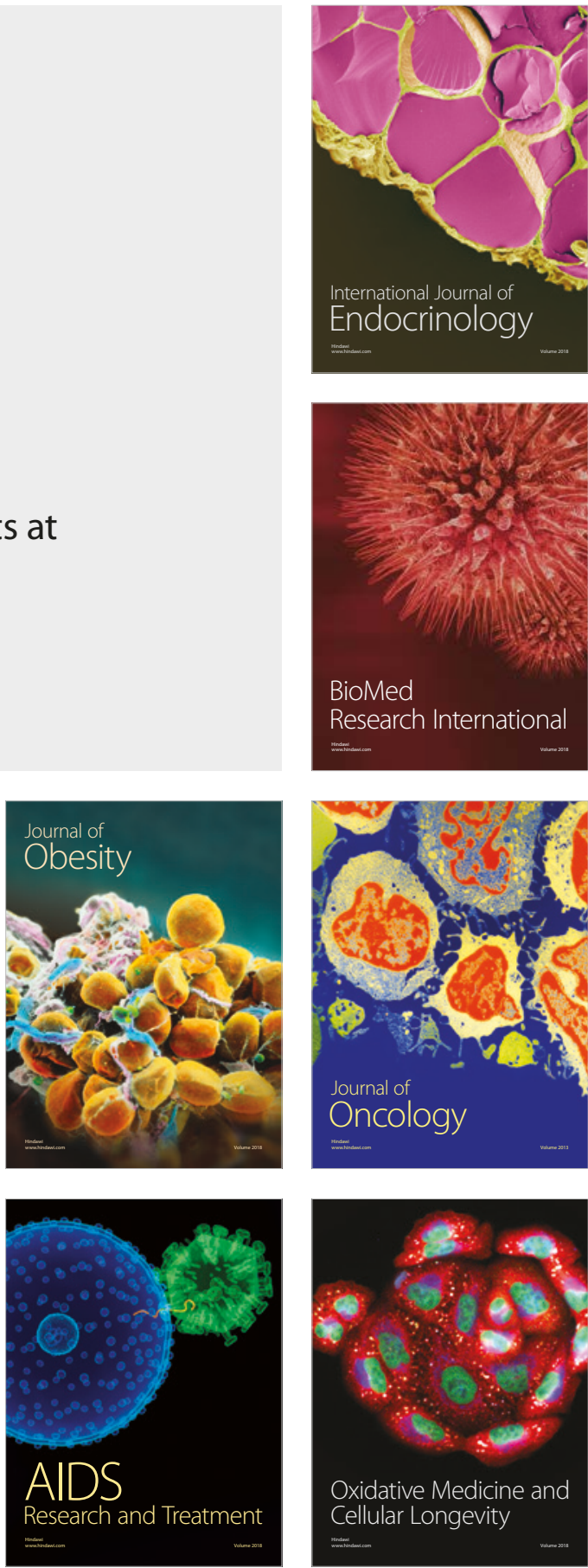